\title{
The main component of an alarm pheromone of kissing bugs plays multiple roles in the cognitive modulation of the escape response
}

\section{Sebastian Minoli ${ }^{+}$, Florencia Palottini ${ }^{\dagger}$ and Gabriel Manrique}

Laboratorio de Fisiología de Insectos, Departamento de Biodiversidad y Biología Experimental, Facultad de Ciencias Exactas y Naturales, Universidad de Buenos Aires, IBBEA, CONICET-UBA, Buenos Aires, Argentina

\section{Edited by:}

Martin Giurfa, Centre National de la Recherche Scientifique - Université Paul Sabatier-Toulouse III, France

\section{Reviewed by:}

Michel Renou, Institut National de la Recherche Agronomique, France

Andrés Arenas, University of

Würzburg, Germany

\section{*Correspondence:}

Sebastian Minoli, Departamento de Biodiversidad y Biología

Experimental, Facultad de Ciencias Exactas y Naturales, Universidad de Buenos Aires, IBBEA,

CONICET-UBA, Ciudad Universitaria,

Pabellón 2, 1428 Buenos Aires,

Argentina

e-mail:minoli@bg.fcen.uba.ar

${ }^{\dagger}$ These authors have contributed equally to this work.
Innate responses in animals can be modulated by experience. Disturbed adults of the triatomine bug Triatoma infestans release an alarm pheromone (AP) that elicits an escape response in conspecific larvae. The main component of this AP, the isobutyric acid (IsoAc), alone has already shown to generate an escape response in this species. However, not much is known about the modulation of this behavior by non-associative and associative cognitive processes. We present here evidences of the cognitive capacities of $T$. infestans larvae in an escape context under different conditioning paradigms, including IsoAc in different roles. We show that: (1) the duration of a pre-exposure to IsoAc plays a main role in determining the type of non-associative learning expressed: short time pre-exposures elicit a sensitization while a longer pre-exposure time triggers a switch from repellence to attractiveness; (2) a simple pre-exposure event is enough to modulate the escape response of larvae to the AP and to its main component: IsoAc; (3) IsoAc and the AP are perceived as different chemical entities; (4) an association between IsoAc and an aversive stimulus can be created under a classical conditioning paradigm; (5) an association between IsoAc and a self-action can be generated under an operant conditioning. These results evince that IsoAc can attain multiple and different cognitive roles in the modulation of the escape response of triatomines and show how cognitive processes can modulate a key behavior for surviving, as it is the escaping response in presence of a potential danger in insects.

Keywords: learning, alarm-pheromone, plasticity, triatomines, associative, non-associative

\section{INTRODUCTION}

Chemical communication in insects is one of the main ways to find sexual partner, aggregate or prevent conspecifics from a danger, among other behaviors. In triatomine bugs (Hemiptera, Reduviidae, Triatominae), adults bear paired exocrine glands in the thorax (metasternal gland) and in the abdomen (Brindley's gland), which are absent in larvae (Brindley, 1930; Schofield and Upton, 1978). It has been proposed for different triatomine species that metasternal gland volatiles mediate sexual communication between adults (Manrique et al., 2006; Crespo and Manrique, 2007; Pontes et al., 2008; Vitta et al., 2009; Zacharias et al., 2010; Manrique and Lorenzo, 2012; Pontes and Lorenzo, 2012). The Brindley's glands, which secrete isobutyric acid (IsoAc) as their main component, are likely to be associated with alarm and defense functions (Kälin and Barrett, 1975; Schofield, 1979; Ward, 1981; Cruz-López et al., 1995; Rojas et al., 2002; Manrique et al., 2006). Particularly in the hematophagous bug Triatoma infestans Klug 1834, the existence of an alarm pheromone (AP) released by disturbed adults that elicits an escape response of conspecific larvae has been reported (Manrique et al., 2006). Moreover, IsoAc alone has also shown to modulate the behavior of this species, being attractive or repellent according to the presented dose (Ward, 1981; Guerenstein and Guerin, 2001).

Although innate responses are essential for surviving, cognitive processes confer individuals the aptitude to acquire or improve skills after a first experience. This capacity varies across species, individuals, and even throughout lifespan and can be modulated by several features of training procedures. Sensitization and habituation are cognitive processes expressed in almost all animals, evincing the adaptive value of these simple forms of plasticity of behavior. Both processes involve nonassociative conditionings, which cause a change in behavior as a result of a first sensorial experience which is not associated to any other cue or reward. While sensitization helps to increase attention to a particular cue (Monteith, 1963; Rakitin et al., 1991; Braun and Bicker, 1992), increasing the probability to find it or avoid it (Rakitin et al., 1991; Hammer et al., 1994; Aggio et al., 1996; Walters et al., 2001; Anderson et al., 2003, 2007; Grubb and Thompson, 2004; Anton et al., 2011; Guerrieri et al., 2012; Minoli et al., 2012), habituation helps to filter out information which is no longer relevant (Duerr and Quinn, 1982). Generally, short exposure times lead mostly to sensitization processes while long exposures provoke an habituation of the response. 
Associative learning is the process by which an association between two stimuli or a behavior and a stimulus is consolidated, if properly reinforced (Bitterman et al., 1983; Heisenberg et al., 1985; Menzel and Muller, 1996). Two main forms of associative learning have been described in animals. In Pavlov's classical conditioning (Pavlov, 1927) a previously neutral stimulus is repeatedly presented together with a reflex eliciting stimuli followed by a reinforcement, until eventually the neutral stimulus will elicit a response on its own. In Skinner's operant conditioning (Skinner, 1937) a certain behavior is followed by a reinforcement, resulting in an altered probability that the behavior will happen again.

Although learning and memory have been widely studied in many insect species, very little has been done to describe the cognitive abilities of hematophagous insects. Host preference or oviposition-sites fidelity of mosquitoes have been shown to be modulated by experience (McCall and Eaton, 2001; McCall et al., 2001; McCall and Kelly, 2002; Alonso et al., 2003; Kaur et al., 2003). However, recent work has been published in which the cognitive capacities of triatomines are evinced. In these works, the authors show that under a classical paradigm, Rhodnius prolixus is able to associate a neutral odor with a positive (Vinauger et al., 2011a) and a negative reinforcement (Vinauger et al., 2011b). Moreover, after a first experience, this species learned to avoid host odors negatively reinforced (Vinauger et al., 2012).

In this work we analyzed the experience-dependent modulation of the escape response of $T$. infestans larvae when confronted to the AP or to its main component, IsoAc. Non-associative protocols were applied to analyze if a chemical pre-exposure may change the escape response of larvae confronted to the AP or the IsoAc. Short and long pre-exposure times were applied to investigate if pre-exposure duration may account for the switch from a sensitization to an habituation process. Crossed preexposure and tests with AP and/or IsoAc were carried out to find out if they are perceived as different chemical entities or not. Classical conditioning of the escape response was studied pairing the IsoAc with a mechanical aversive stimulus and then measuring changes in the response to IsoAc. Operant conditioning was analyzed in a spatial preference paradigm by training individuals to avoid one zone of an experimental arena by delivering IsoAc every time they entered such zone and measuring then changes in their spatial preference caused by this training. We present clear evidences of the cognitive modulation of the escape response in triatomines under all forms of learning tested. We discuss the multiple modulatory roles of the main component of the AP, IsoAc, in different cognitive processes.

\section{MATERIALS AND METHODS}

Larvae of $T$. infestans were reared in the laboratory insectary at $28 \pm 2{ }^{\circ} \mathrm{C}$ temperature and $60 \pm 20 \%$ relative humidity, under a 12:12 h L/D illumination regime. All instars were fed weekly on live hens handled according to the biosafety rules from the Servicio de Higiene y Seguridad of the Facultad de Ciencias Exactas y Naturales, Universidad de Buenos Aires. For the assays, 15-25 days old non-fed fourth instar larvae were used. A total of 1600 larvae were used along this work. Insects were used only once and then discarded.

All experiments (training and test procedures, see below) were carried out during the first hours of the scotophase (i.e., $1-5 \mathrm{~h}$ after lights were turned-off) as to match the maximal activity period observed for these insects (Lazzari, 1992). Experiments were performed under total darkness conditions to match the phase of photoperiod in which animals were tested (i.e., scotophase) and at the same time to avoid the possible utilization of visual cues by larvae. The temperature of the experimental room was set to $25 \pm 1^{\circ} \mathrm{C}$ before the beginning of each assay with an electric fan heater, which was turned-off before the start of the experiments. The room's relative humidity range was $40 \pm 10 \%$.

\section{EXPERIMENT 1. NON-ASSOCIATIVE LEARNING: CHEMICAL PRE-EXPOSURE}

We applied different pre-exposure protocols using different odors and times, and we analyzed if this chemical stimulation could modify the escape response of larvae of $T$. infestans confronted to the same or different odors and/or doses.

\section{TRAINING PROCEDURES}

Pre-exposure to different chemical stimuli was carried out using an acrylic cylindrical flask (Figure 1A, $6 \mathrm{~cm}$ height, $2.5 \mathrm{~cm}$ diameter) divided horizontally by a plastic mesh $(0.5 \mathrm{~mm}$ pore $)$. Larvae were placed over the mesh and the different stimuli sources at the bottom of the flask. In this way, individuals were exposed to the volatiles released by either one disturbed adult (AP) or a rectangular piece of filter paper $(2 \times 1 \mathrm{~cm})$ loaded with $10 \mu \mathrm{g}$ of IsoAc (i.e., approximate content of Brindley's glands, Palottini, pers. communication).

Pre-exposure to IsoAc was achieved either for a short (1 min, Figure 1: P1) or a long period (60 min, Figure 1: P2). For the short pre-exposure, $50 \mu \mathrm{l}$ of dichloromethane (DCM) containing $10 \mu \mathrm{g}$ of IsoAc were loaded on the filter piece of paper and inserted at the bottom of the pre-exposure flask. One larva was then placed over the plastic mesh for $1 \mathrm{~min}$. For the long preexposure a similar procedure was performed but larvae were left over the mesh during $60 \mathrm{~min}$. The stimulus-loaded filter paper (i.e., $50 \mu \mathrm{l}$ of DCM with $10 \mu \mathrm{g}$ of IsoAc) was changed for a new reloaded one every 15 min (i.e., four equally loaded pieces of paper) to ensure the presence of the odor in the air along time. Control assays were carried out by loading the solvent alone (i.e., $50 \mu \mathrm{l}$ of DCM) in the filter paper (naive insects).

Pre-exposure to the AP was carried out by placing one adult in the lower part of the flask. Insects were allowed to climb onto a piece of filter paper, which was subsequently placed into the preexposure flask to avoid disturbing them. Once inside the flask, the stimulus-adult was artificially disturbed by grabbing one of its legs with forceps during $30 \mathrm{~s}$. One larva was placed over the plastic mesh during $1 \mathrm{~min}$ of pre-exposure to the released blend. Control assays were carried out by leaving the adult undisturbed (naive insects).

After pre-exposure protocols, animals were transferred to individual acrylic cylindrical flasks $(3 \times 2.5 \mathrm{~cm})$ (Figure 1B) in darkness for $10 \mathrm{~min}$ before the beginning of the tests in a dual-choice olfactometer (Figure 1C, see below). 


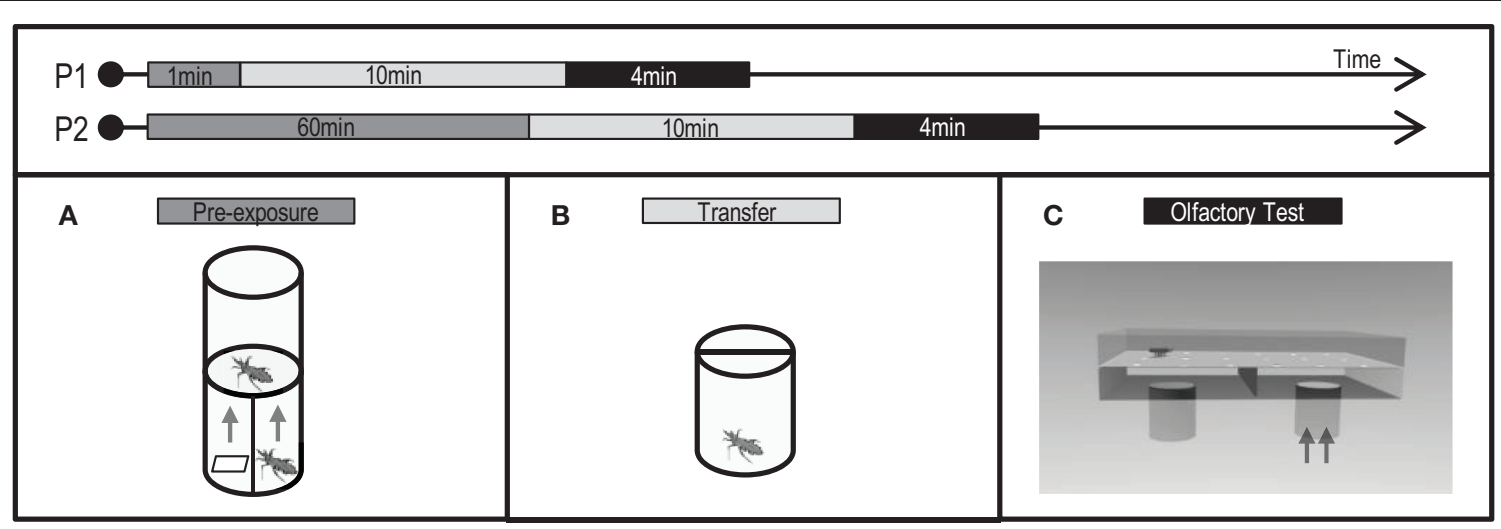

FIGURE 1 | Protocols (P1: short pre-exposure, P2: long pre-exposure) applied and experimental devices used to analyze the effects of a non-associative exposure in the escape response of $T$. infestans larvae.
Chemical pre-exposure was made stimulating with the alarm pheromone (AP) or with its main component, isobutyric acid (IsoAc). Pre-exposure flask (A), transfer flask (B), and test arena (C).

\section{MEASUREMENT OF THE ESCAPE RESPONSE IN A DUAL-CHOICE OLFACTOMETER}

The escape response elicited by the AP of T. infestans adults or by IsoAc was tested using a dual-choice walking olfactometer in absence of air currents (Figure 1C). The arena consisted of a rectangular acrylic box $(15 \times 10 \times 4 \mathrm{~cm})$ with holes on the floor ( $2 \mathrm{~mm}$ diameter), attached to a sub-chamber divided in two equal parts by an odor-impermeable transversal acrylic plate, interconnected to independent lower openings into which removable flasks $(10 \mathrm{ml})$ containing the stimuli were attached.

Either an undisturbed or a disturbed T. infestans adult or different doses of IsoAc $(0.1,10$, or $1000 \mu \mathrm{g})$ in $50 \mu \mathrm{l}$ of DCM, or $50 \mu \mathrm{l}$ of DCM alone loaded on a piece of filter paper $(2.5 \times$ $0.5 \mathrm{~cm}$ ) were placed in the stimulus-flasks. In this way, stimuli (the volatiles emitted by disturbed or undisturbed adults or by the loaded piece of paper) entered by diffusion into each of the two parts of the sub-chamber independently and continued to diffuse up to the arena through the holes, creating a chemical gradient over it. A Kraft paper with holes matching the floor holes served as substrate for the experimental larvae and avoided possible chemical contamination between assays, as it was changed every time a new bug was released.

In each individual assay, one flask was used as control (either an undisturbed adult or a DCM loaded filter paper was inserted) and the other was set as stimulus (i.e., a disturbed adult or IsoAc was inserted). Both flasks were attached to the sub-chamber and one larva was then placed in the middle of the arena and left covered with an inversed flask over it during 1 min for context familiarization and odor diffusion. The larva was then released by gently lifting the covering flask and its behavior was registered during 4 min by means of a video-camera connected to a digital recorder. Control assays were performed adding only DCM into both flasks. The position of the stimulus was randomly alternated between assays.

The time spent in each side of the arena was registered as a measure of the olfactory preference of insects. A preference index $(\mathrm{PI})$ ranging from -1 to 1 was calculated as $P I=\left(T_{C}-T_{S}\right) /\left(T_{C}+T_{S}\right)$, where $T_{C}$ is the time (in seconds) spent in the control side of the arena and $\mathrm{T}_{S}$ the time spent in the side where the stimulus was added. In control series (i.e., no stimulus added) $T_{S}$ is the time in seconds spent in one side of the arena chosen randomly. PIs near $-1,0$, or 1 indicate repellence, random distribution, or attraction to the added stimulus, respectively. Deviations from a random distribution (i.e., $P I=$ 0 ) of the larvae over the arena were assessed by means of One Sample $T$-Tests. Differences between pre-exposures were assessed by means of One Way ANOVAs followed by Tuckey post-hoc comparisons when needed. A total of 40 replicates were achieved for each treatment.

\section{RESULTS 1A: MODULATION OF THE ESCAPE RESPONSE TO IsOAc AFTER A SHORT OR A LONG PRE-EXPOSURE}

The main component of the AP of adult T. infestans, IsoAc, has already shown to repel adults of the same species (Ward, 1981). Here, we show that naive larvae are repelled by $10 \mu \mathrm{g}$ of IsoAc (Figure 2A; One Sample T-Test, $p=0.04$ ) and not by other doses ( $p>0.05$ for all cases). A similar but sharper dosedependent avoidance was obtained after a short pre-exposure of $1 \mathrm{~min}$ to $10 \mu \mathrm{g}$ of IsoAc (Figure 2B; One Sample $T$-Test, $p=$ 0.001 for $10 \mu \mathrm{g}$ IsoAc, $p>0.05$ for other doses). A long exposure $(60 \mathrm{~m})$ to IsoAc resulted in a dramatic change from repellence to attractiveness to $10 \mu \mathrm{g}$ of IsoAc (Figure 2C; One Sample T-Test, $p=0.00005$ ).

To quantify the effect of pre-exposure we compared the intensity of the escape response of naive and short pre-exposed larvae elicited by $10 \mu \mathrm{g}$ of IsoAc. We found a significantly higher repellence after a short pre-exposure to IsoAc (Figures 2A,B; T-Test, $p=0.0002$; comparison showed with a vertical line).

\section{RESULTS 1B: COGNITIVE DISCRIMINATION BETWEEN AP AND IsOAc}

We analyze here the lineal- and cross-effects of a brief chemical pre-exposure to the AP (i.e., a blend released by disturbed adults) or to its main component alone (IsoAc) in the escape response generated by the same two odors. As expected, all groups of larvae avoided the side of the arena containing $10 \mu \mathrm{g}$ of IsoAc (Figure 3, black dots; One Sample T-Test, 


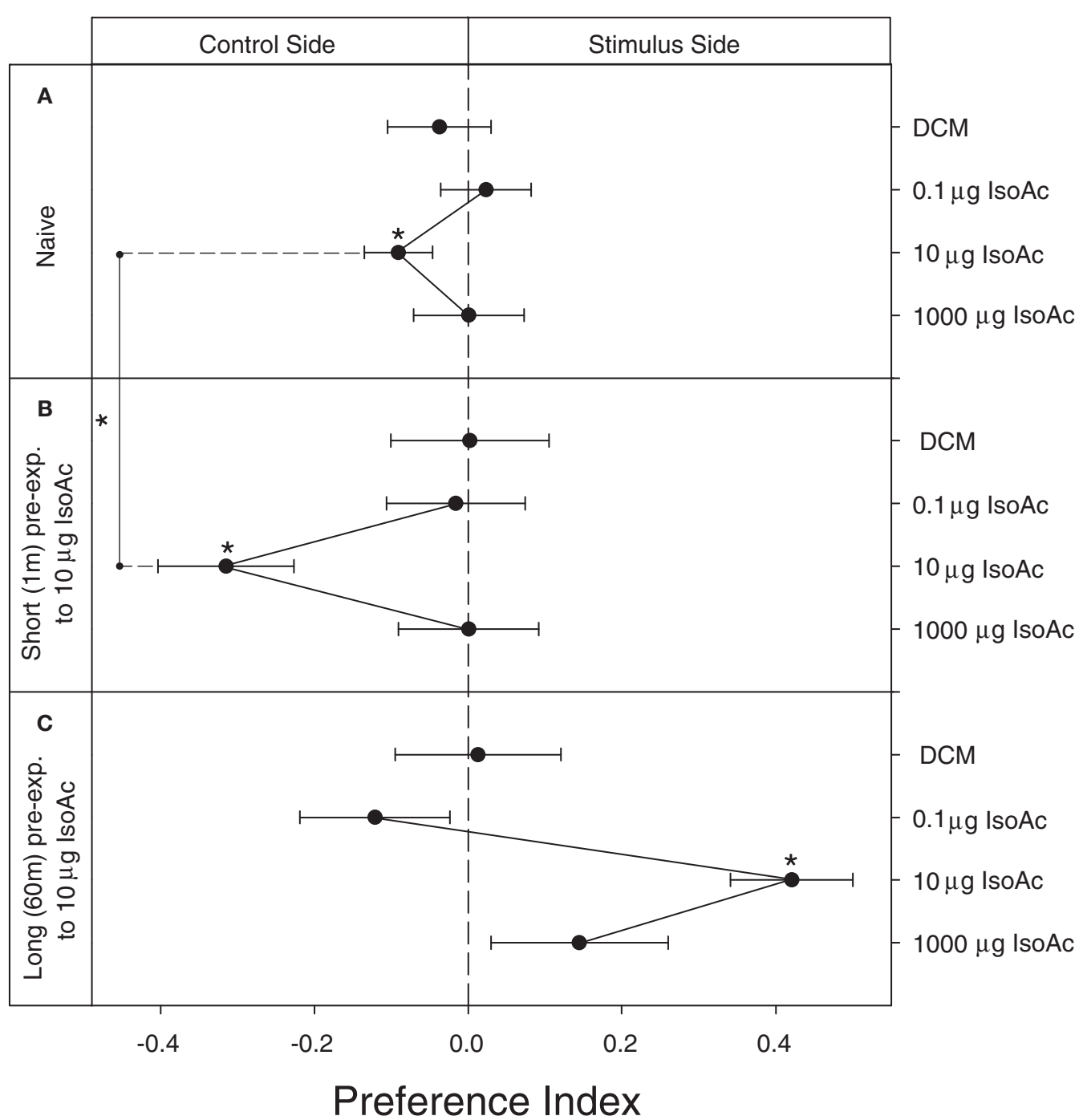

FIGURE 2 | Experiment 1A: Modulation of the escape response to IsoAc after a non-associative pre-exposure to the same compound. (A) Naive, (B) short pre-exposed $(1 \mathrm{~m})$, and (C) long pre-exposed $(60 \mathrm{~m})$ larvae. $N=40$ assays for each dose. Asterisks show significant differences from a $\mathrm{Pl}=0$ (One Sample T-Test, $p<0.05)$. Asterisk over the vertical line show differences between treatments ( $T$-Test, $p<0.05)$. Naive larvae escaped from $10 \mu \mathrm{g}$ of IsoAc (A). A clear sensitization effect after a short pre-exposure was evident (B). A long pre-exposure protocol inversed the significance of the IsoAc (C). $p<0.05$, for three cases). Similarly, naive- and IsoAc-exposed larvae avoided the AP (Figure 3, white triangles; One Sample $T$-Test, $p<0.05$, for both cases). Only the larvae pre-exposed to AP did not avoid the AP during test (One Sample T-Test, $p>0.05)$.

When we compared the effect of pre-exposure, we found that the escape response to the AP did not vary with a chemical experience (Figure 3; white triangles; One Way ANOVA, $p>0.05$, significant differences shown with different numbers). Conversely, a significant effect of pre-exposure to IsoAc over the escape response of larvae was revealed (black dots; One Way ANOVA, $p=0.04)$. Post-hoc comparisons showed that a brief pre-exposure to $10 \mu \mathrm{g}$ of IsoAc increased the escape response to the same compound as compared to naive larvae (Tuckey, $p=0.03$, significant differences shown with different letters) but a pre-exposure to AP did not (Tuckey, $p>0.05)$.

\section{EXPERIMENT 2. ASSOCIATIVE LEARNING: CLASSICAL CONDITIONING}

A classical conditioning arrangement was designed for T. infestans larvae in which the negative hedonic value of IsoAc was expected to increase by associating the presence of this aversive compound with a mechanical disturbance. In posterior tests, larvae should increase their avoidance behavior against IsoAc.

\section{TRAINING PROCEDURES}

Training was carried out by placing a larva inside a closed glass flask (Figure 4A, $5 \mathrm{~cm}$ height, $3 \mathrm{~cm}$ diameter) with one inputtube ( $5 \mathrm{~mm}$ diameter) bearing a clean air current, into which 


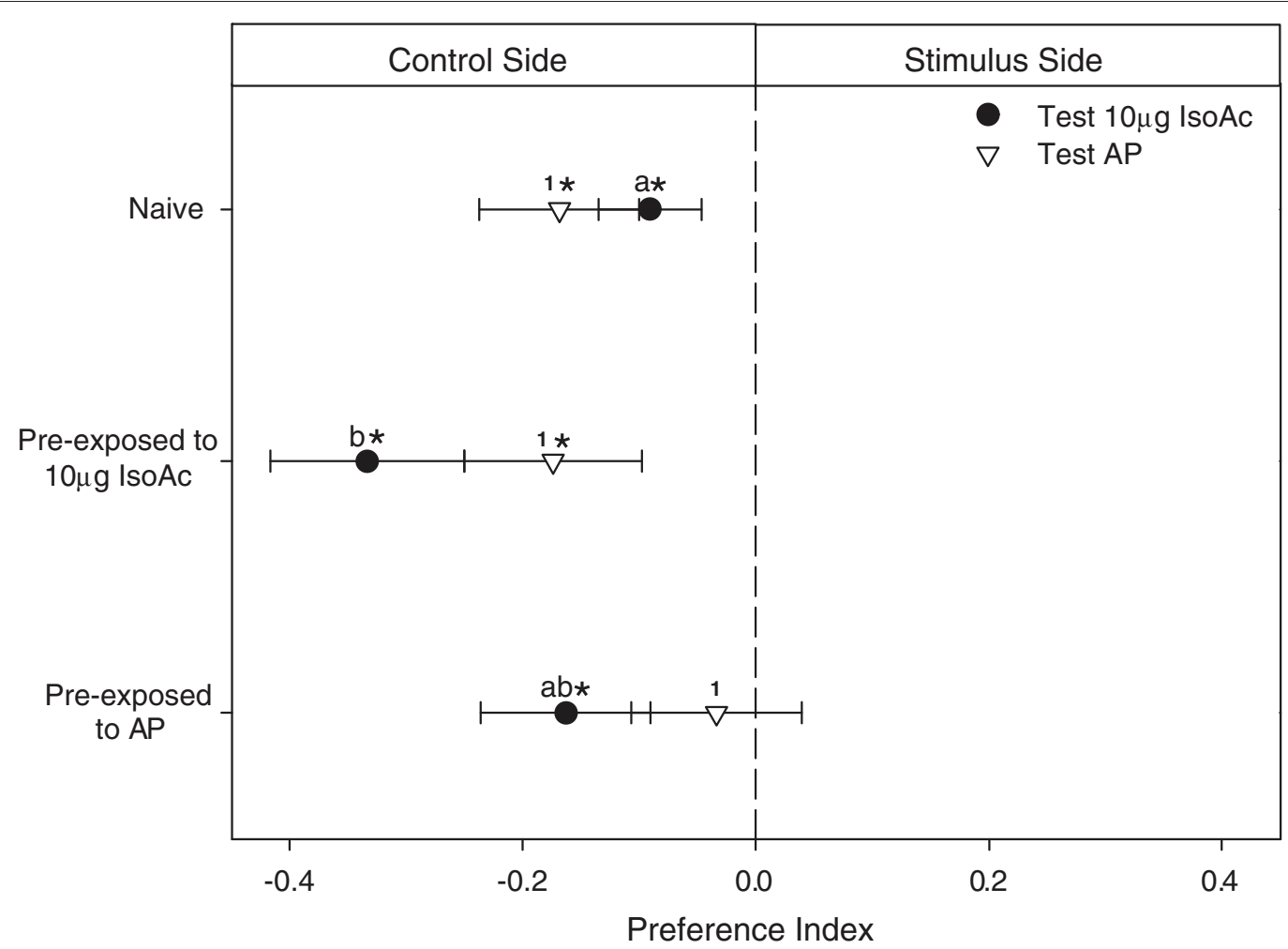

FIGURE 3 | Experiment 1B: Modulation of the escape response of larvae to IsoAc or to the AP after a non-associative pre-exposure to IsoAc or to the AP. $N=40$ assays for each treatment. Asterisks show significant differences from a $P I=0$ (One Sample $T$-Test, $p<0.05)$. Different letters or numbers show significant differences between treatments ( $T$-Test, $p<0.05$ ). Naive larvae avoided both, $10 \mu \mathrm{g}$ of IsoAc and the AP. Pre-exposure to IsoAc increased the escape response to the same compound but not to the AP. Pre-exposure to the AP did not change the innate escape response to IsoAc nor to AP.

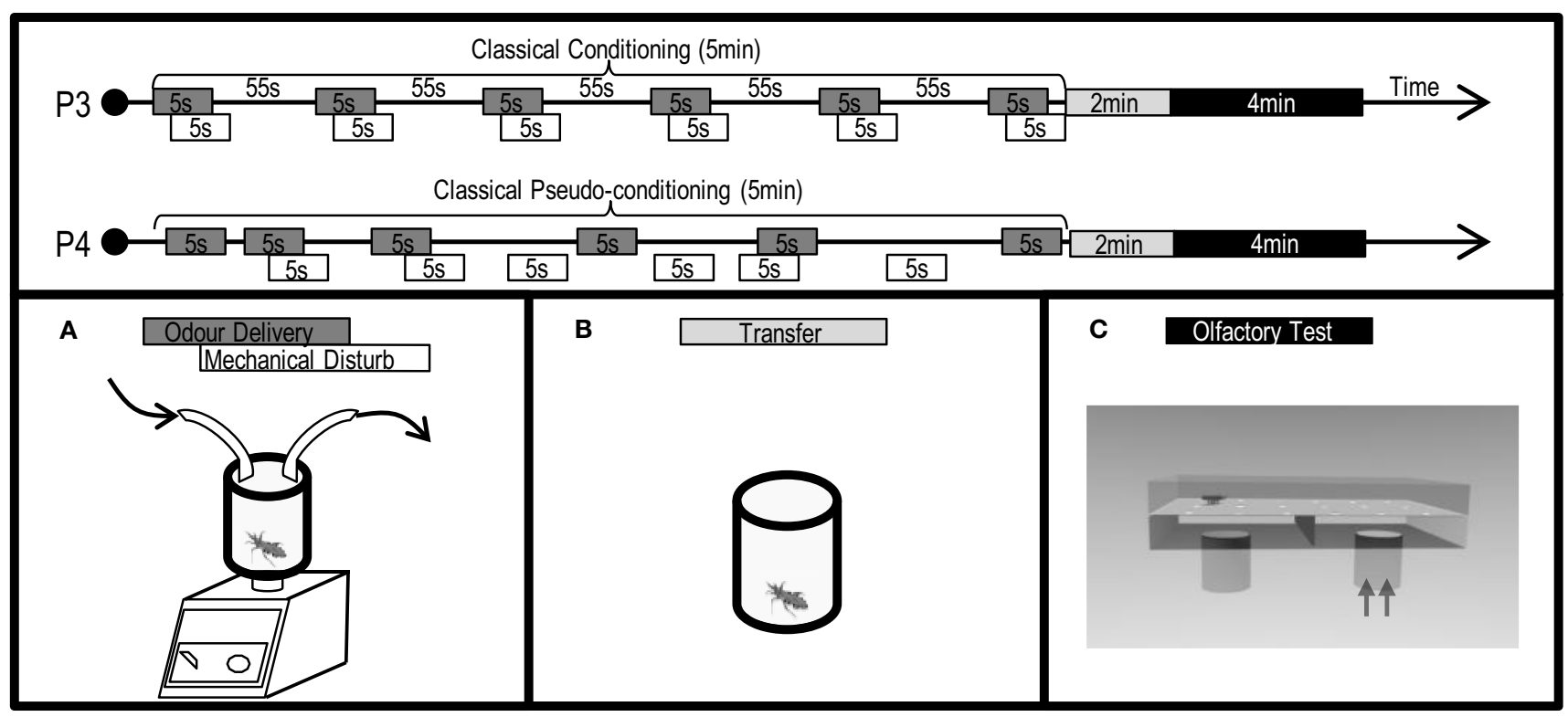

FIGURE 4 | Protocols (P3: conditioning, P4: pseudo-conditioning) applied and experimental devices used to analyze the effects of an associative classical conditioning in the escape response of $\boldsymbol{T}$. infestans larvae promoted by isobutyric acid (IsoAc). Training flask (A), transfer flask (B), and test arena (C). 
controlled doses of IsoAc could be added manually with an electronic switch by interposing a loaded piece of filter paper (10 or $1000 \mu \mathrm{g}$ of IsoAc), and one output-tube conducting odors to an external extractor. The flask containing the larva was placed over a mixer (Vortex $1000 \mathrm{rpm}$ ) that generated a mechanical disturbance electronically controlled. The timing of the IsoAc delivery and the mechanical disturbance caused by the mixer was manipulated as to generate different learning protocols.

Conditioning assays (Figure 4: P3) consisted of 5 min trainings in which larvae perceived once every minute a trial, pairing a 5-s puff of IsoAc (10 or $1000 \mu \mathrm{g}$ ) with a 5-s mechanical disturbance, shifted $2 \mathrm{~s}$ forward (i.e., 6 trials, inter trial interval $53 \mathrm{~s}$ ). A pseudoconditioning protocol (Figure 4: P4) was performed in which 6 puffs of IsoAc and 6 mechanical disturbances were delivered in a random manner (i.e., not paired). Insects in the naive group were not submitted to IsoAc nor to the mechanical disturbance.

After training, animals were transferred to individual acrylic cylindrical flasks $(3 \times 2.5 \mathrm{~cm})$ (Figure 4B) in darkness for $2 \mathrm{~min}$ before the beginning of the tests in a dual-choice olfactometer (Figure 4C).

\section{MEASUREMENT OF THE ESCAPE RESPONSE IN A DUAL-CHOICE OLFACTOMETER}

The escape response triggered by IsoAc was tested using the dualchoice walking olfactometer in absence of air currents described above (Figure 4C). Two doses of IsoAc were loaded on the filter paper matching the dose used during training: 10 or $1000 \mu \mathrm{g}$. For each test, the time spent in each side of the arena by each larva was registered during $4 \mathrm{~min}$. The PI was calculated as described before (see Measurement of the Escape Response in a Dual-Choice Olfactometer, for more details). Deviations from a random distribution (i.e., $P I=0$ ) were assessed for each treatment by means of One Sample $T$-Tests. A total of 40 replicates were achieved for each treatment.

\section{RESULTS: MODULATION OF THE ESCAPE RESPONSE TO IsOAc AFTER A CLASSICAL ASSOCIATION WITH A MECHANICAL DISTURBANCE}

In these assays we applied a classical conditioning pairing the delivery of a puff of IsoAc with a mechanical disturbance. Naive larvae presented (as shown before) an innate escape response to $10 \mu \mathrm{g}$ of IsoAc (Figure 5A; One Sample T-test, $p=0.04$ ). Although this repellent effect was expected to increase by a training in which the hedonic value of IsoAc $10 \mu \mathrm{g}$ was supposed to become more negative after an association with a second aversive stimulus (i.e., the mechanical disturbance), no escape response at all was observed for the conditioning group (Figure 5A; One Sample $T$-test, $p>0.05$ ). As the pseudo-conditioning assays with $10 \mu \mathrm{g}$ of IsoAc (in which the delivery of the puff of IsoAc was not paired in time with the mechanical disturbance) also resulted in a lack of response to $10 \mu \mathrm{g}$ during tests (Figure 5A; One Sample $T$-test, $p>0.05$ ), the vanishing of the escape response might then be an effect of habituation to the IsoAc delivered during training.

When $1000 \mu \mathrm{g}$ of IsoAc was used during training, even if naive animals did not avoid the zone with this dose of IsoAc (Figure 5B; One Sample T-test, $p>0.05$ ), once submitted to a conditioning in which we paired $1000 \mu \mathrm{g}$ of IsoAc with the mechanical disturbance, larvae started to avoid it (Figure 5B; One Sample T-test, $p=0.02)$. In their corresponding pseudo-conditioning assays, no

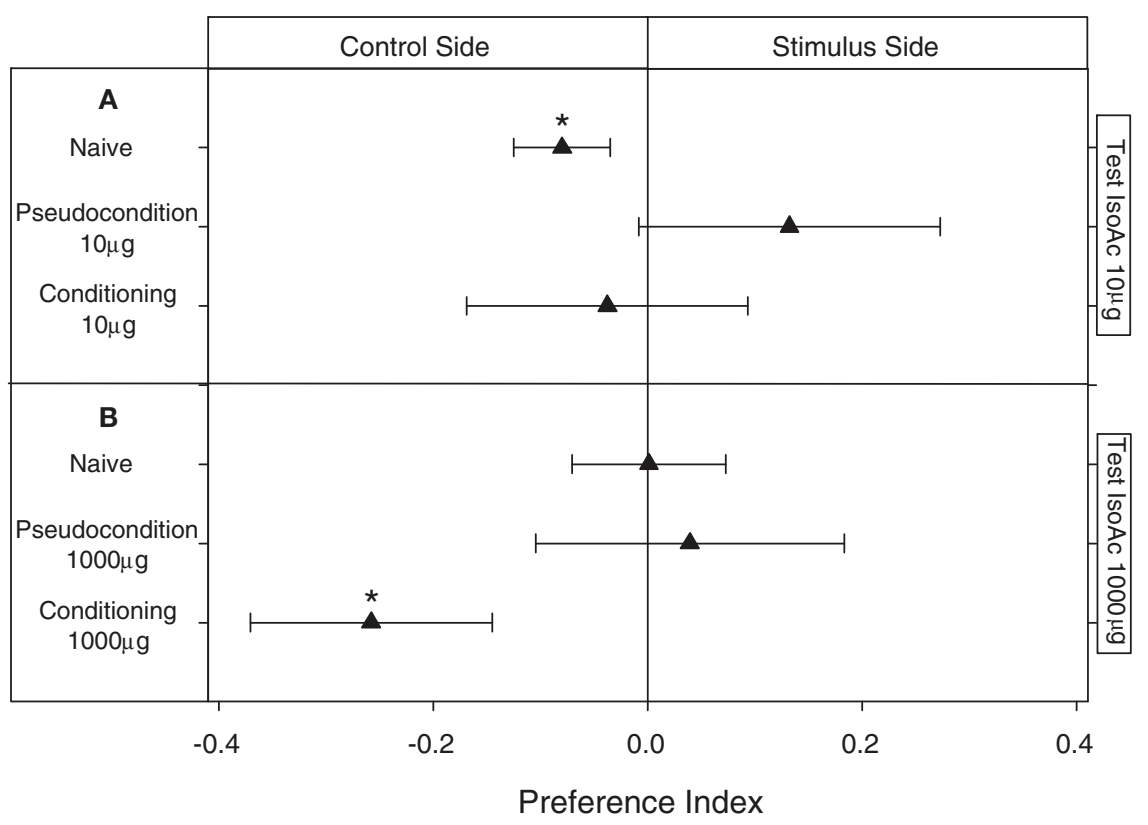

FIGURE 5 | Experiment 2: Modulation of the escape response of larvae to IsoAc after an associative classical conditioning training. (A) Training and test with $10 \mu \mathrm{g}$ of IsoAc. (B) Training and test with $1000 \mu \mathrm{g}$ of IsoAc. $N=40$ assays for each treatment. Asterisks show significant differences from a $P I=0$ (One Sample $T$-Test, $p<0.05$ ). No changes in the escape response were observed after an aversive conditioning with $10 \mu \mathrm{g}$ of IsoAc (A). However, although not-trained animals (naive or pseudo-conditioning groups) did not avoid $1000 \mu \mathrm{g}$ of IsoAc, after an associative training they were strongly repelled by this dose (B). 
escape response was registered (Figure 5B; One Sample $T$-test, $p>0.05)$. These results show that the lack of escape response presented by larvae when $1000 \mu \mathrm{g}$ of IsoAc was presented in one side of the arena (Experiment 1A, Figure 2) is not a constraint of the setup (e.g., due to a chemical homogenization of the arena caused by high doses), but instead a result of the insects' preference, as in this section larvae avoided the same dose.

\section{EXPERIMENT 3. ASSOCIATIVE LEARNING: OPERANT CONDITIONING}

An operant conditioning training was designed in which larvae of $T$. infestans were trained to avoid one half of an experimental arena (predetermined as punished side) by delivering IsoAc whenever they entered this zone during training. During posterior tests without odors delivered, larvae were expected to avoid the side where the IsoAc was delivered during training.

\section{TRAINING PROCEDURES}

Training was performed using a rectangular experimental arena $(8 \times 5 \times 4 \mathrm{~cm})$ with half of its floor covered with smooth paper and the other half with rough paper (Figure 6A). A silicon tube connected to a triangular flat diffusor delivered continuously either a clean $(50 \mu \mathrm{l}$ of DCM) or a IsoAc loaded air current to the whole arena, at the floor level. To deliver the IsoAc to the insects, 10 or $1000 \mu \mathrm{g}$ of IsoAc in $50 \mu \mathrm{l}$ of DCM were loaded on a filter paper $(2.5 \times 0.5 \mathrm{~cm})$ and interposed in the clean air current. Before each assay, one side of the arena (and so one texture) was settled as the punished side and the other as the safe side in a pseudo-random manner (i.e., randomly but balanced along 40 replicates). The timing of the IsoAc delivery was defined by the position of each larva in conditioning assays (Figure 6: P5) or manipulated by the experimenter in pseudo-conditioning assays (Figure 6: P6).

For conditioning assays, one larva was released in the middle of the arena and, during 5 min of training, IsoAc was added to the current whenever the larva entered the previously determined punished side. Pseudo-conditioning assays were performed in which IsoAc was added to the current independently from the position of the larva over the arena during $5 \mathrm{~min}$. The total IsoAc time delivered during pseudo-conditioning assays was calculated from conditioning series. Two doses of IsoAc were used during trainings: 10 or $1000 \mu \mathrm{g}$.

After training, animals were transferred to individual acrylic cylindrical flasks $(3 \times 2.5 \mathrm{~cm})$ (Figure 6B) in darkness for $1 \mathrm{~min}$ before the beginning of the odorless tests (Figure 6C).

\section{MEASUREMENT OF THE SPATIAL PREFERENCE IN AN EXPERIMENTAL ARENA WITHOUT ODORS}

The spatial preference (guided by the texture of the floor) was registered over the experimental arena used during training (Figure 6C) but without the addition of IsoAc to the air current (i.e., a continuous current bearing only $50 \mu \mathrm{l}$ of DCM).

In each assay, one larva was placed in the middle of the arena covered with an inversed flask. After 1 min of context familiarization the cover was gently lifted releasing the larva. Its behavior was then registered using a video camera connected to a digital recorder.

The time spent in each side of the arena was registered during conditioning ( $5 \mathrm{~min}$ ) and tests $(5 \mathrm{~min})$. A PI ranging from -1 to 1 was calculated as $P I=\left(T_{S}-T_{P}\right) /\left(T_{S}+T_{P}\right)$, where $T_{S}$ is the time (in seconds) spent in the safe side of the arena and $T_{P}$ the time spent in the punished side. PIs near $-1,0$, or 1 indicate preference to stay in the punished side, random distribution or preference to stay in the safe side of the arena, respectively. Deviations from a random distribution (i.e., $P I=0$ ) of the larvae over the arena were assessed by means of One Sample T-Tests. A total of 40 replicates were achieved for each treatment.

\section{RESULTS: AVOIDANCE OF A NEUTRAL ZONE AFTER AN OPERANT ASSOCIATION WITH IsOAC}

An operant conditioning paradigm was applied by delivering a punishment (puff with IsoAc) whenever larvae entered the punished side of the arena. Differently from previous experiments, this protocol allowed us to calculate the PIs already during

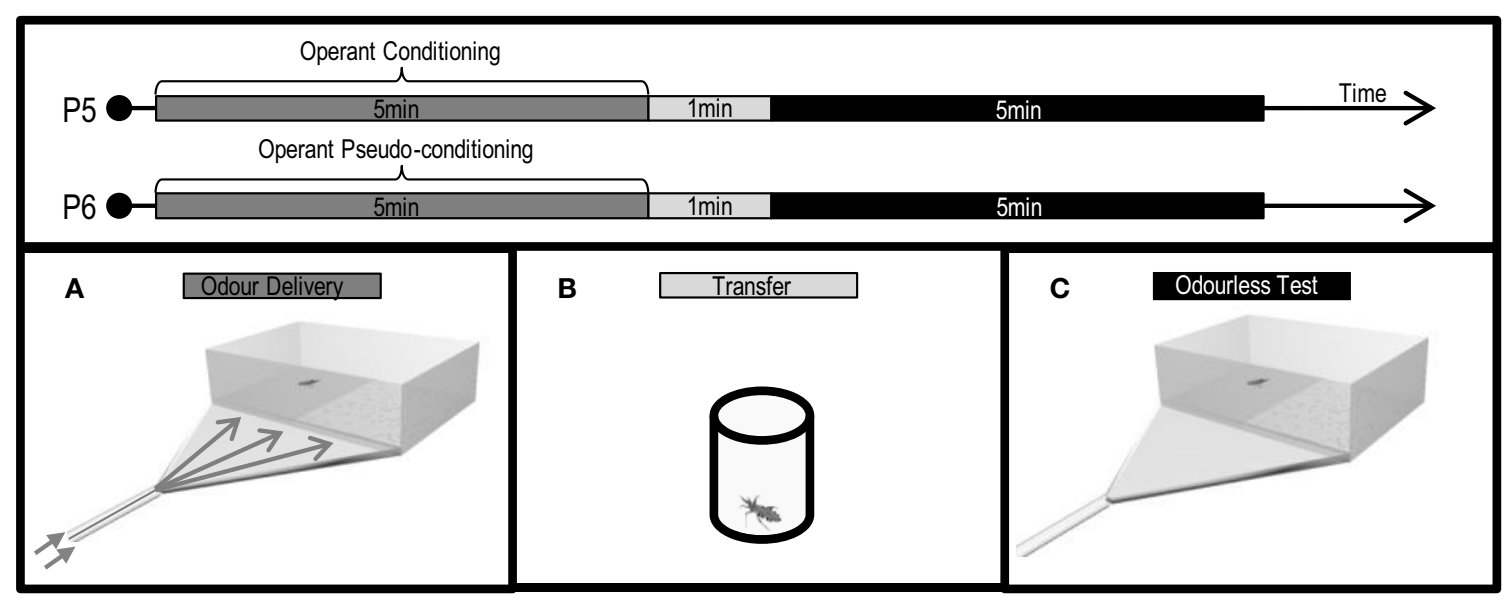

FIGURE 6 | Protocols (P5: conditioning, P6: pseudo-conditioning) applied and experimental devices used to analyze the effects of an associative operant conditioning in the escape response of $\boldsymbol{T}$. infestans larvae promoted by isobutyric acid (IsoAc). Training arena (A), transfer flask (B), and test arena (C). 
trainings. We found that when $10 \mu \mathrm{g}$ of IsoAc was used as negative reinforcement, although a trend to avoid the punished side was observed during both, training and test, no statistical differences were observed from a random distribution (Figure 7A; One Sample $T$-test, $p>0.05$, for both cases). But, when $1000 \mu \mathrm{g}$ of IsoAc were used as punishment, a significant repellence was observed already during training (Figure 7B; One Sample T-test, $p=0.01$ ). After a transfer event of $1 \mathrm{~min}$ and even without odordelivery during tests, larvae continued to avoid the side previously defined as punished (Figure 7B; One Sample T-test, $p=0.009$ ).

Larvae stimulated with either 10 or $1000 \mu \mathrm{g}$ of IsoAc independently from their position in the arena (i.e., pseudo-conditioning series) presented a random distribution during both, training and tests (Figures 7A,B; One Sample T-test, $p>0.05$ for all cases).

\section{DISCUSSION}

All forms of learning tested here showed to modulate the escape response of $T$. infestans to IsoAc. Although a highly relevant behavior for survival (as it is escaping from a potential danger) generally requires a genetic basis, it was shown here that it is strongly modulated by experience in many ways. The sensitization elicited after a brief perception of the IsoAc was reflected in an increased attention to this compound and an increased proneness to respond. In natural environments, being more sensitive to an AP, if it has been recently and briefly perceived (i.e., meaning that the danger is probably still around), can be the cue to perform a rapid escape and so survive.

Although an habituation expressed as a lack of response was expected after a long pre-exposure to IsoAc, we found instead an inversion of the significance of the signal, becoming attractive for larvae. IsoAc has been already reported by other authors to be repellent at high doses and to become attractive at low doses (Ward, 1981; Guerenstein and Guerin, 2001). Besides being released by Brindley's glands, IsoAc is a constituent of human sweat (Cork and Park, 1996) and probably of other triatomine hosts. However, the amount of this compound release by vertebrate skin is much lower as compared to the AP content. It might happen then that the attraction of long-pre-exposed larvae to a normally repellent dose of IsoAc $(10 \mu \mathrm{g})$ was a result of a sub-estimation of the real dose sensed due to an habituation process. Nevertheless, other alternative processes such as sensory priming might also explain the attraction after a long-term exposition (Schacter and Buckner, 1998).

We can discard that the results observed were a case of sensory adaptation after a pre-exposure to IsoAc, as we found either an increased or an inversed response to IsoAc, but never a lack of response, as it would be expected if a sensory adaptation was to occur. Nor an increase in response nor an inversion of sense of the response (i.e., meaning that there is still a response) can be explained by a sensory adaptation. Similarly, motor fatigue is discarded as a decrease in the locomotor response would also cause a lack of response.

Duration of pre-exposure specifies the sign of the nonassociative cognitive modulation: short pre-exposure elicits sensitization and long pre-exposure seems to elicit habituation. Although it was not the objective of this work, we think there might be some point somewhere between short and long pre-exposure duration in which sensitization and habituation

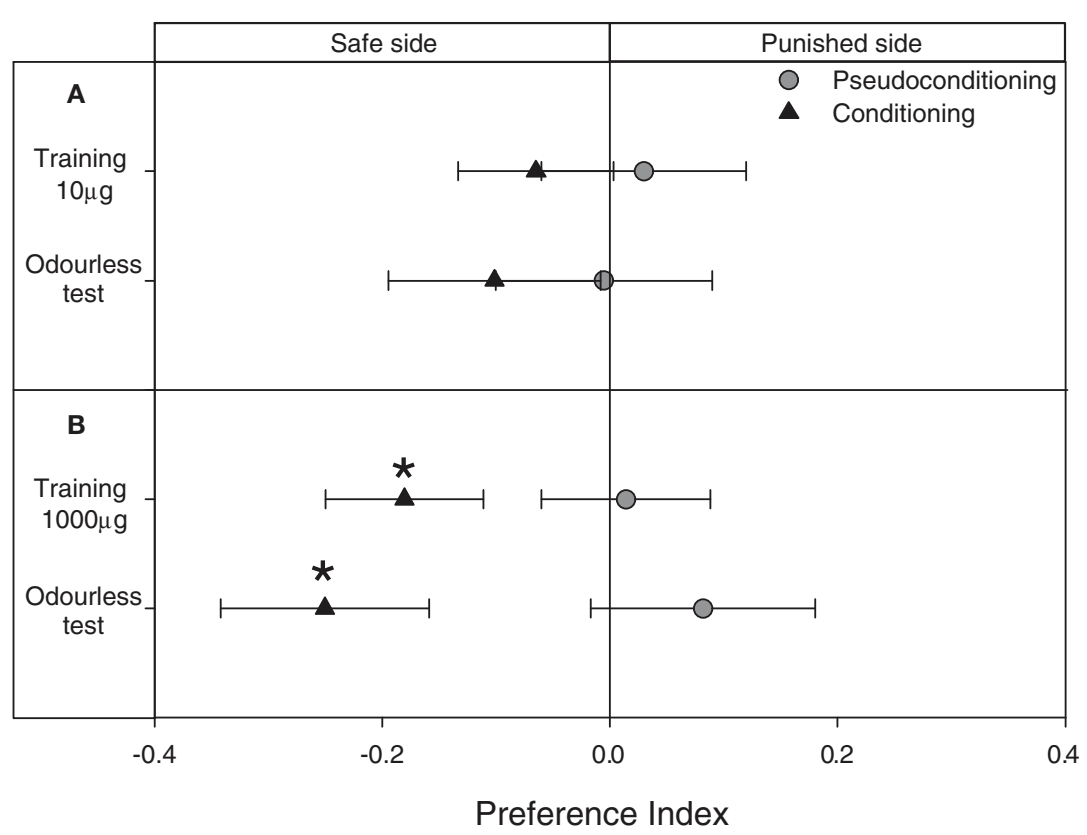

FIGURE 7 | Experiment 3: Modulation of the escape response of larvae to IsoAc after an associative operant conditioning training. (A) Training with $10 \mu \mathrm{g}$ of IsoAc. (B) Training with $1000 \mu \mathrm{g}$ of IsoAc. $N=40$ assays for each treatment. Asterisks show significant differences from a $\mathrm{Pl}=0$ (One
Sample $T$-Test, $p<0.05)$. A not-significant trend to avoid the punished side was observed when $10 \mu \mathrm{g}$ IsoAc was used as punishment during training (A). When $1000 \mu \mathrm{g}$ of IsoAc were used during training, larvae learned to avoid the punished side and continued to avoid it during odorless tests (B). 
might be hidden one behind the other. It's surprising to realize that different exposure times to the same compound can generate such opposite effects. Based in our results we cannot inform whether physiological mechanisms involved in both processes are independent or not.

We show in this work that a pre-exposure to IsoAc can modulate the sensitivity to the same compound in T. infestans larvae by revealing changes in their escape response when confronted to different doses after a short or a long pre-exposure. However, the relevance of the dose of IsoAc used for pre-exposure was not studied. We chose here to carry out all pre-treatments by exposing larvae to a biologically relevant dose: $10 \mu \mathrm{g}$ of IsoAc, which is similar to the quantity present in the glands, estimated by quantitative gas chromatography (Palottini, pers. communication) and elicited an innate response in our experimental setup. Further studies can be focused on analyzing if pre-exposure effect is modulated by concentration and if the switch from sensitization to habituation might be modulated not only by pre-exposure duration but also by pre-exposure concentration. Additionally, we do not know yet if pre-exposure effect is doses dependent, i.e., if pre-exposure to a certain dose only elicits a change in sensitivity to the same dose or to other also. In this work we only found a modulation of the escape response when larvae were tested to the same dose at which they were exposed.

As mentioned previously, IsoAc is the main component of the AP emitted by mechanically disturbed T. infestans adults (Manrique et al., 2006). Although pre-exposure to a biologically relevant dose of IsoAc increases the response of bugs to the same compound, it does not elicit a cognitive plasticity to the AP blend. The opposite is also valid, as pre-exposure to AP did not modulate the response to IsoAc. These results suggest that when an individual perceives the AP blend, it is not just the main component the responsible for the escape response, but instead a combination with other volatile compounds released during disturbance. However, it might occur that IsoAc alone is perceived as a complete different chemical entity when presented in a background of other compounds, as it is the case when the AP is perceived by these bugs. Further studies are necessary to analyze this question.

IsoAc per se is innately perceived by $T$. infestans larvae as an aversive stimulus. Applying classical conditioning protocols, the negative hedonic value of IsoAc was increased by associating it with a second aversive stimulus: a mechanical disturbance. Mechanical disturbance was applied as aversive stimulus as it can mimic the reaction to a predator or a defensive host bothered by triatomines. For this reason, the increase in the escape

\section{REFERENCES}

Aggio, J., Rakitin, A., and Maldonado, H. (1996). Serotonin-induced short- and long-term sensitization in the crab Chasmagnathus. Pharmacol. Biochem. Behav. 53, 441-448. doi: 10.1016/0091-3057 (95)02015-2

Alonso, W. J., Wyatt, T. D., and Kelly, D. W. (2003). Are vectors able to learn about their hosts. A case study with Aedes aegypti mosquitoes.

response of larvae after the co-occurrence of the contingency IsoAc/mechanical disturbance shows how the correct association between these two stimuli might be relevant for survival. Larvae might in this way increase the negative hedonic value of the AP if a potential danger becomes more real (i.e., sensing a more direct risk indicator as a mechanical vibration).

In the operant experiments, we tested if the delivery of IsoAc could act as punishment for triatomine larvae. To our knowledge, this is the first report in which an aversive odor is used as negative reinforcement in animals. IsoAc per se, although perceived as an aversive stimulus, is not the real danger but instead the warning of the presence of a potential danger (e.g., predator or defensive host). In these experiments larvae learned to avoid zones with higher presence of IsoAc. This learned behavior might allow triatomines to avoid zones where previously high concentrations of IsoAc were present, i.e., zones with higher probabilities to find a potential danger.

For both associative protocols applied here (i.e., classical and operant conditioning), higher concentrations of the chemical stimulus seemed to better consolidate the association presented. It is well described that salience of stimuli is a key parameter for a better memory consolidation in many animals (Pelz et al., 1997). Here, even if better escape responses were obtained by loading $10 \mu \mathrm{g}$ of IsoAc in the stimulus piece of paper, the best memory scores were obtained when $1000 \mu \mathrm{g}$ of IsoAc were used for both, classical and operant conditioning protocols.

We show in this report that a particular compound, IsoAc, can attain different roles in the cognitive modulation of a particular behavior. First of all, IsoAc may act as an unconditioned stimulus (US), as it generates an innate escape response (i.e., an unconditioned response) in these insects. We showed also, in Experiment 1 , that IsoAc can act as sensitization agent when delivered in short puffs. Moreover, a long pre-exposure to this molecule can even inverse its biological significance for triatomines, suggesting and habituation process involved. Additionally, in Experiment 2 we show that IsoAc can act as conditioned stimulus (CS) under a classical paradigm. Finally, in Experiment 3 IsoAc takes the role of a negative reinforcement in an operant conditioning. As far as we know this is the first work in which the existence of a single molecule having so many and different cognitive roles is reported.

\section{ACKNOWLEDGMENTS}

The authors are deeply indebted to Martin Giurfa for fruitful discussions and suggestions. This investigation received financial support from ANPCyT (PICT PIDRI 0029-2009), CONICET, and UNIVERSIDAD de BUENOS AIRES.

Anderson, P., Sadek, M. M., and Hansson, B. S. (2003). Preexposure modulates attraction to sex pheromone in a moth. Chem. Senses 28, 285-291. doi: 10.1093/chemse/28.4.285

Anton, S., Evengaard, K., Barrozo, R. B., Anderson, P., and Skals, N. (2011). Brief predator sound exposure elicits behavioral and neuronal longterm sensitization in the olfactory system of an insect. Proc. Natl. Acad.
Sci. U.S.A. 108, 3401-3405. doi: 10.1073/pnas.1008840108

Bitterman, M. E., Menzel, R., Fietz, A., and Schafer, S. (1983). Classical conditioning of proboscis extension in honeybees (Apis mellifera). J. Comp. Psychol. 97, 107-119. doi: 10.1037/0735-7036.97.2.107

Braun, G., and Bicker, G. (1992). Habituation of an appetitive reflex in the honeybee. J. Neurophysiol. 67, 588-598. 
Brindley, M. D. H. (1930). On the metasternal scent-glands of certain Heteroptera. Trans. R. Entomol. Soc. Lond. 78, 199-208. doi: 10.1111/j.13652311.1930.tb00383.x

Cork, A., and Park, K. C. (1996). Identification of electrophysiologically-active compounds for the malaria mosquito, Anopheles gambiae, in human sweat extracts. Med. Vet. Entomol. 10, 269-276. doi: 10.1111/j.13652915.1996.tb00742.x

Crespo, J. G., and Manrique, G. (2007). Mating behavior of the hematophagous bug Triatoma infestans: role of brindley's and metasternal glands. J. Insect Physiol. 53, 708-714. doi: 10.1016/j.jinsphys.2007.03.014

Cruz-López, L., Morgan, E. D., and Ondarza, R. N. (1995). Brindley's gland exocrine products of Triatoma infestans. Med. Vet. Entomol. 9, 403-406. doi: 10.1111/j.13652915.1995.tb00013.x

Duerr, J. S., and Quinn, W. G. (1982). Three Drosophila mutations that block associative learning also affect habituation and sensitization. Proc. Natl. Acad. Sci. U.S.A. 79, 3646-3650. doi: 10.1073/pnas.79.11.3646

Grubb, M. S., and Thompson, I. D. (2004). The influence of early experience on the development of sensory systems. Curr. Opin. Neurobiol. 14, 503-512. doi: 10.1016/j.conb.2004.06.006

Guerenstein, P. G., and Guerin, P. M. (2001). Olfactory and behavioural responses of the blood-sucking bug Triatoma infestans to odours of vertebrate hosts. J. Exp. Biol. 204, 585-597.

Guerrieri, F., Gemeno, C., Monsempes, C., Anton, S., Jacquin-Joly, E., Lucas, P., et al. (2012). Experience-dependent modulation of antennal sensitivity and input to antennal lobes in male moths (Spodoptera littoralis) preexposed to sex pheromone. J. Exp. Biol. 215, 2334-2341. doi: 10.1242/ jeb.060988

Hammer, M., Braun, G., and Mauelshagen, J. (1994). Foodinduced arousal and non-associative learning in honeybees: dependence of sensitization on the application site and duration of food stimulation. Behav. Neural Biol. 62, 210-223. doi: 10.1016/S0163-1047(05)80019-6

Heisenberg, M., Borst, A., Wagner, S., and Byers, D. (1985). Drosophila mushroom body mutants are deficient in olfactory learning.
J. Neurogenet. 2, 1-30. doi: 10.3109/01677068509100140

Kälin, M., and Barrett, F. M. (1975). Observations on the anatomy, histology, release site, and function of Brindley's glands in the bloodsucking bug, Rhodnius prolixus (Heteroptera: Reduviidae). Ann. Entomol. Soc. Am. 68, 126-134.

Kaur, J. S., Lai, Y. L., and Giger, A. D. (2003). Learning and memory in the mosquito Aedes aegypti shown by conditioning against oviposition deterrence. Med. Vet. Entomol. 17, 457-460. doi: 10.1111/j.13652915.2003.00455.x

Lazzari, C. R. (1992). Circadian organization of locomotion activity in the haematophagous bug Triatoma infestans. J. Insect Physiol. 38, 895-903. doi: 10.1016/0022-1910(92)90101-I

Manrique, G., and Lorenzo, M. G. (2012). The sexual behavior of Chagas' Disease vectors: chemical signals mediating communication between male and female Triatomine bugs. Psyche 2012, 8. doi: $10.1155 / 2012 / 862891$

Manrique, G., Vitta, A., Ferreira, R., Zani, C., Unelius, C., Lazzari, C., et al. (2006). Chemical communication in Chagas Disease vectors. Source, identity, and potential function of volatiles released by the Metasternal and Brindley's glands of Triatoma infestans adults. J. Chem. Ecol. 32, 2035-2052. doi: 10.1007/s10886006-9127-7

McCall, P. J., and Eaton, G. (2001). Olfactory memory in the mosquito Culex quinquefasciatus. Med. Vet. Entomol. 15, 197-203. doi: 10.1046/j.0269-283x.2001.00304.x

McCall, P. J., and Kelly, D. W. (2002). Learning and memory in disease vectors. Trends Parasitol. 18, 429-433. doi: 10.1016/S1471-4922(02)02370-X

McCall, P. J., Mosha, F. W., Njunwa, K. J., and Sherlock, K. (2001). Evidence for memorized site-fidelity in Anopheles arabiensis. Trans. R. Soc. Trop. Med. Hyg. 95, 587-590. doi: 10.1016/S0035-9203(01)90087-2

Menzel, R., and Muller, U. (1996). Learning and memory in honeybees: from behavior to neural substrates. Аnпu. Rev. Neurosci. 19, 379-404. doi: 10.1146/ annurev.ne.19.030196.002115

Minoli, S., Kauer, I., Colson, V., Party, V., Renou, M., Anderson, P., et al. (2012). Brief exposure to sensory cues elicits stimulusnonspecific general sensitization in an insect. PLoS ONE 7:e34141. doi: 10.1371/journal.pone.0034141
Monteith, L. G. (1963). Habituation and associative learning in Drino bohemica Mesn. (Diptera: Tachinidae). Can. Entomol. 95, 418-426. doi: 10.4039/Ent95418-4

Pavlov, I. P. (1927). Lectures on Conditioned Reflexes. New York, NY: International Publishers.

Pelz, C., Gerber, B., and Menzel, R. (1997). Odorant intensity as a determinant for olfactory conditioning in honeybees: roles in discrimination, overshadowing and memory consolidation. J. Exp. Biol. 200, 837-847.

Pontes, G., Bohman, B., Unelius, C. R., and Lorenzo, M. (2008). Metasternal gland volatiles and sexual communication in the triatomine bug, Rhodnius prolixus. J. Chem. Ecol. 34, 450-457. doi: 10.1007/s10886-008-9431-5

Pontes, G., and Lorenzo, M. G. (2012). Female metasternal gland odors mediate male aggregation in Rhodnius prolixus, a triatomid bug. Med. Vet. Entomol. 26, 33-36. doi: 10.1111/j.1365-2915.2011.00983.x

Rakitin, A., Tomsic, D., and Maldonado, H. (1991). Habituation and sensitization to an electrical shock in the crab Chasmagnathus. Effect of background illumination. Physiol. Behav. 50, 477-487. doi 10.1016/0031-9384(91)90533-T

Rojas, J. C., Rios-Candelaria, E. Cruz-Lopez, L., Santiesteban, A. Bond-Compean, J. G., Brindis, Y., et al. (2002). A reinvestigation of Brindley's gland exocrine compounds of Rhodnius prolixus (Hemiptera: Reduviidae). J. Med. Entomol. 39, 256-265. doi 10.1603/0022-2585-39.2.256

Schacter, D. L., and Buckner, R. L. (1998). Priming and the Brain. Neuron 20, 185-195. doi: 10.1016/S0896-6273(00)80448-1

Schofield, C., and Upton, C. P. (1978). Brindley's scent-glands and the metasternal scent-glands of Panstrongylus megistus (Hemiptera, Reduviidae, Triatominae). Rev. Bras. Biol. 38, 665-678.

Schofield, C. J. (1979). Demonstration of isobutyric acid in some triatomine bugs. Acta Trop. 36, 103-105.

Skinner, B. F. (1937). Two types of conditioned reflex: a reply to Konorski and Miller. J. Gen. Psychol. 16, 272-279. doi 10.1080/00221309.1937.9917951

Vinauger, C., Buratti, L., and Lazzari, C. R. (2011a) Learning the way to blood: first evidence of dual olfactory conditioning in a bloodsucking insect, Rhodnius prolixus. I. Appetitive learning. J. Exp. Biol. 214, 3032-3038. doi: 10.1242/jeb.056697
Vinauger, C., Buratti, L., and Lazzari, C. R. (2011b) Learning the way to blood: first evidence of dual olfactory conditioning in a bloodsucking insect, Rhodnius prolixus. II. Aversive learning. J. Exp. Biol. 214 3039-3045. doi: 10.1242/jeb.057075

Vinauger, C., Pereira, M. H., and Lazzari, C. R. (2012). Learned host preference in a Chagas disease vector, Rhodnius prolixus. Acta Trop. 122, 24-28. doi: 10.1016/j.actatropica.2011.11.007

Vitta, A., Bohman, B., Unelius, C., and Lorenzo, M. (2009). Behavioral and electrophysiological responses of Triatoma brasiliensis males to volatiles produced in the Metasternal glands of females. J. Chem. Ecol. 35, 1212-1221. doi: 10.1007/s10886-009-9709-2

Walters, E., Illich, P., Weeks, J., and Lewin, M. (2001). Defensive responses of larval Manduca sexta and their sensitization by noxious stimuli in the laboratory and field. J. Exp. Biol. 204, 457-469.

Ward, J. P. (1981). A comparison of the behavioural responses of the haematophagous bug, Triatoma infestans to synthetic homologues of two naturally occurring chemicals (n- and isobutyric acid). Physiol. Entomol. 6, 325-329. doi: 10.1111/j.13653032.1981.tb00277.x

Zacharias, C., Pontes, G., Lorenzo, M. and Manrique, G. (2010). Flight initiation by male Rhodnius prolixus is promoted by female odors. J. Chem. Ecol. 36, 449-451. doi: 10.1007/s10886-010-9779-1

Conflict of Interest Statement: The authors declare that the research was conducted in the absence of any commercial or financial relationships that could be construed as a potential conflict of interest.

Received: 24 May 2013; accepted: 14 June 2013; published online: 05 July 2013. Citation: Minoli S, Palottini F and Manrique G (2013) The main component of an alarm pheromone of kissing bugs plays multiple roles in the cognitive modulation of the escape response. Front. Behav. Neurosci. 7:77. doi: 10.3389/ fnbeh.2013.00077

Copyright (๑) 2013 Minoli, Palottini and Manrique. This is an open-access article distributed under the terms of the Creative Commons Attribution License, which permits use, distribution and reproduction in other forums, provided the original authors and source are credited and subject to any copyright notices concerning any third-party graphics etc. 